\title{
Lehrwerkauswahl für die sprachpraktischen Übungen der Primarschullehrerausbildung \\ oder: \\ Kann der Unterricht auch ohne ein Lehrwerk erfolgreich sein?
}

\author{
Alice Brychová
}

Ein richtiges Unterrichtsmaterial auszuwählen, ist eine der wichtigsten Voraussetzungen des erfolgreichen Unterrichts. Das Lehrwerk bildet nämlich die Basis für die methodische Konzeption der Unterrichtseinheiten, es beeinflusst in vielfältigen Beziehungen alle Faktoren, die den Unterricht bestimmen. Natürlich hängt die Gestaltung des Unterrichts auch von anderen Faktoren ab, aber das benutzte Lehrwerk bestimmt, ob dem Lehrer viel Freiraum überlassen bleibt oder ob jeder Unterrichtsschritt ganz genau festgelegt ist (vgl. BIMMEL/ KAST/NEUNER: 2003, 6).

Heutzutage ist das Angebot an Unterrichtsmaterialien für Deutsch als Fremdsprache relativ groß, nur die richtige Entscheidung zu treffen, das bleibt vielleicht deswegen eine noch schwierigere Aufgabe für jeden Lehrer selbst. Diese Aufgabe kann erleichtert werden, wenn der Unterrichtende seine Studierenden oder Schüler genau kennt und wenn er im Voraus für sich und seine Lerner klare Entscheidungskriterien formuliert. Das Lehrwerk kann nämlich als Träger vieler Informationen verbalen und nonverbalen Charakters emotional und kognitiv auf den Lerner (und wahrscheinlich auch auf den Lehrer) wirken und seine Erwartungen an den Unterricht aber auch seine späteren Erinnerungen an den Unterricht und generell seine Vorstellungen davon, was Fremdsprachenunterricht eigentlich bedeutet, stark beeinflussen (vgl. BRYCHOVÁ: 2007, 1).

In neueren methodischen Konzeptionen stehen die LernerimZentrum des Lernens und Lehrens (das Prinzip der Lernerzentriertheit und der Erfahrungsorientierung) und deswegen werden sie in allen Phasen des Unterrichts zu Mitgestaltern des Unterrichts. Lerner sollten auch über den gesamten Lernprozess mitbestimmen und mitentscheiden können. Dies bedeutet auch über: Lernziele, Methoden, Lernmaterialien, Lernzeit, Lernorte, Lernpartner bis hin zur Evaluation. Sie sollten also auch das Recht haben über das benutzte Material mitzuentscheiden oder sogar selbst einige Übungsmaterialien für ihre Mitschüler zu entwerfen.

Das Lehrwerk sollte dann als ein Angebot für die eigentliche Unterrichtsgestaltung verstanden werden. Weitere Faktoren, die die Organisation des Unterrichts beeinflussen, sind das geltende Curriculum und der damit verbundene Lehrplan, die eigenen Vorstellungen des Lehrers von einem guten Unterricht und auch diejenigen der Schüler. Außer den Vorstellungen der Lernenden spielen hier auch die Lerner 
selbst eine Rolle, ihre Lerngewohnheiten und -erfahrungen, ihr Vorwissen, natürlich auch ihre Einstellung gegenüber dem Fach, in unserem Fall der deutschen Sprache als Fremdsprache.

\section{Die Lehrwerkauswahl in der Primarschullehrerausbildung an der Pädagogischen Fakultät in Brünn}

Natürlich bemühen wir uns auch immer das geeignete Lehrwerk für die sprachpraktischen Übungen unserer Student/-innen im Studienprogramm Lehramt für die Primarschule auszuwählen. Vor allem sollte das Material methodisch gut durchdacht sein und die Spezifika unserer Lernenden berücksichtigen. Es muss sich v. a. um ein modernes Material handeln, das den zukünftigen Lehrern/-innen Lern- und Unterrichtsformen präsentiert, die sowohl zum Erfolg führen, als auch ein Erlebnis beim Lernen ermöglichen, das Spaß mit Lernerfolg verbindet. Die Unterrichtsformen, die die Studierenden selbst im Unterricht erleben, beeinflussen nach unserer Meinung nämlich die Entwicklung des methodischen Denkens der zukünftigen Lehrer/-innen in dem Maße, dass die methodische Vielfalt in der Studienzeit später zu einer ebensolchen Vielfalt im eigenen Unterricht der ehemaligen Studenten beitragen kann. Für viele Lehrer/-innen gilt nämlich im übertragenen Sinne, dass der "Geist“ ihres eigenen Lehrers „hinter der Tafel steht“, wenn sie später selber unterrichten. Deswegen möchten wir ein aktuelles und modernes Lehrwerk benutzen. Solche modernen Lehrwerke werden mit folgenden Attributen charakterisiert: „der Aufbau ist transparent, das didaktische Konzept ist offen und flexibel und lernerorientiert" (NEUNER u. a.: 1988, in Deutsch aktiv neu). In vielen neueren Lehr- und Lernmaterialien schlägt sich außerdem die Tendenz nieder, selbstreflektiertes Lernen selbst zum Unterrichtsthema zu machen. Reflexionen über Lernziele, Lernertypen und Lernstrategien sowie Übungen zum „Lernen des Lernens“ (metakognitives Lernen) und Anregungen zum selbstentdeckenden Lernen haben daher ihren festen Platz in vielen modernen Lehrwerken. Diese Themen sind für den genannten sprachpraktischen Unterricht natürlich auch von großer Bedeutung.

Vor drei Jahren haben wir unter den Studierenden des Lehramts für die Primarschule im Rahmen der Unterstützung ihrer Mitverantwortung bei der Entscheidung über das geeignete Lehrwerk für die sprachpraktischen Übungen vom ersten bis zum sechsten Semester eine Umfrage durchgeführt. Die Ergebnisse zeigten eindeutig, dass sich die Studierenden in der Mehrheit für ein Lehrwerk tschechischer Autoren entschieden, das zwar kommunikativ orientiert ist, aber das nicht immer authentische Texte präsentiert und stark auf die Automatisierung der deduktiv präsentierten Grammatik abzielt. ${ }^{1}$

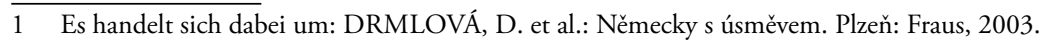

Brünner Hefte zu Deutsch als Fremdsprache • Jahrgang 1・Nummmer 1/2 • 2008 
Bis zu diesem Zeitpunkt hatten wir versucht, den oben genannten Unterricht mit dem Lehrwerk em-Brückenkurs (bzw. em-Hauptkursim letzten Semester) zu realisieren. Es handelt sich um ein modernes Lehrwerk, das vor allem Fertigkeiten trainiert und auch induktive Grammatikvermittlung stark unterstützt. Die Erfahrungen, die wir und vor allem unsere Studierenden mit dem em-Lehrwerk gemacht haben, waren jedoch ziemlich enttäuschend. Die meisten Studenten/-innen hielten die Orientierung in dem Lehrwerk für schwierig und den Aufbau einzelner Lektionen für unübersichtlich und ungewöhnlich, obwohl jede Seite ihr eigenes didaktisches Thema behandelt, das explizit formuliert wird. Die Organisation des Lehrwerkes ist so, dass jeder Fertigkeit eine Seite gewidmet wird und Teilkompetenzen als Mittel für das sprachliche Handeln präsentiert werden. Das manifestiert sich z. B. an der Präsentation der neuen Grammatik oder auch des Wortschatzes anhand von Texten. Vielleicht auch deswegen fanden viele Studenten gerade die Texte zu schwierig, weil sie authentische Sprache verwenden und teilweise mit unbekanntem Wortschatz arbeiten, der dann im Arbeitsbuch nach Wortarten geordnet, nicht aber übersetzt wird. Die Semantisierung des Wortschatzes sollte vom Kontext der genannten Texte aktiv von Lernern entdeckt werden. Ferner kamen die Studierenden auch deswegen nicht mit den Texten zu Recht, weil sie nicht gewohnt waren, die Texte gegebenenfalls nur global oder selektiv zu verstehen und den Wortschatz direkt aus den Texten zu lernen anstatt über auswendig zu lernende Vokabellisten. Wir bemerkten auch, dass es für die Studenten sehr schwierig war, in Texten Ironie oder Mehrdeutigkeit zu erkennen ${ }^{1}$ oder auch nur Humor als solchen zu bemerken. Das mag auch mit der, in der Mittelschule aufgebauten Erwartungshaltungen zusammen hängen, dass ein Lehrbuchtext immer ernst ist. Das Lehrwerk em-Brückenkurs, der erste Teil einer dreiteiligen Reihe, ist nämlich für Lerner bestimmt, die sich am Ende der oberen Grundstufe befinden, und sollte ihnen als Brücke zur Mittelstufe dienen. In der Terminologie des GERRs bedeutet dies, dass das Lehrbuch die Lerner, die sich bereits auf dem Niveau B1 befinden, an die Niveaustufe B2 heranführen sollte. Diese Stufe hielten wir für das erste Semester für adäquat, weil wir damals noch davon überzeugt waren, dass die Kenntnisse und Fertigkeiten der Absolventen einer Mittelschule mindestens im Bereich zwischen diesen Niveaustufen angesiedelt seien. Außerdem vermuteten wir, dass die meisten Interessenten für das Studium der deutschen Sprache im Lehramt für die Primarstufe Absolventen des Abiturs in Deutsch sein werden, dessen Schwierigkeitsgrad auch dem Niveau B1 bzw. B2 entsprechen sollte. Obwohl die meisten Studierenden tatsächlich erfolgreiche Absolventen des Abiturs waren, schätzten sich die meisten von ihnen anhand des am Lehrstuhl für deutsche Sprache und Literatur verwendeten Sprachenportfolios eher im Bereich der Niveaustufe A2 ein. Dies war für uns ein Signal dafür, dass

1 In den Deskriptoren des Europäischen Sprachenportfolios und in den Kann-Beschreibungen im GERR gehört allerdings die Fähigkeit Ironie, Satire, Mehrdeutigkeit in Texten zu erkennen zu den komplexen Fähigkeiten auf dem Niveau C2. 
das Abiturniveau den Standardbeschreibungen nicht immer entspricht. Andere Studierende waren sogar noch wirkliche Anfänger.

Unsere Argumente für die Wahl des Arbeitsmaterials sind dabei immer gleich geblieben: Es sollte ein möglichst fertigkeits- und lernerorientiertes Lehrwerk sein, das eine Progression bis zum Sprachniveau B2 aufweist. Die Erfahrungen zeigten jedoch, dass das Ausgangsniveau fast Anfängerniveau sein sollte, weil viele Studierende auch gravierende Wissenslücken in den Grundkenntnissen aufwiesen, und es gerade die Grundkenntnisse der Sprache sind, die sie später den Schülern in ihrer Unterrichtspraxis auf der Primarstufe vermitteln sollen.

In dieser Situation haben die meisten Studenten und Studentinnen unter den möglichen Lehrwerken, die derzeit auf dem tschechischen aber auch auf dem deutschen Markt zu finden sind, das Lehrwerk Německy s úsmèvem nově ausgewählt. Dieses Lehrwerk wurde im Jahre 2003 neu gestaltet. Die ursprüngliche Variante wurde aber schon in den 90er Jahren, nach der politischen Wende, herausgegeben und wird im Vergleich zu anderen damals benutzten Lehrwerken als innovativ angesehen. Den Vorteil dieses Lehrwerks sehen unsere Studierenden wahrscheinlich darin, dass es sich um einen Band handelt, der die Lerner eigentlich von Null-Kenntnissen bis zum Niveau B2 (wie die Herausgeber versprechen) hinführen kann. Es handelt sich um ein Lehrwerk, das für die relevante Alters- und Sozialgruppe konzipiert wurde, d. h. für Hochschulstudenten. Es enthält viele Themen, die besonders junge Studierende ansprechen sollen, wie z. B. der Stundenplan, mein Deutsch-und Fremdsprachenunterricht, Auslandspraktikum oder auch Partys und Ferien. Wirglauben, dass unsere Studenten und Studentinnen das Lehrwerk auch deswegen bevorzugten, weil es eine für sie übersichtliche Organisation aufwies. Trotz ihres jugendlichen Alters sind unsere Studierenden nämlich bereits sehr stark von der Textorganisation in ihren älteren Lehrbüchern und durch eine gewisse traditionsmäßige Lernkultur des tschechischen Milieus beeinflusst. Wahrscheinlich sind sie gewohnt, dass die Unterrichtszeit eher den lehrerzentrierten und stark gesteuerten Aufgaben gehört und das eigene selbstständige Lernen erst in der individuellen Hausarbeit stattfindet. In den Lehrwerken der deutschen Verlage, die wir auch mit der genannten Lernergruppe ausprobiert haben, hatten sie - obwohl sich in diesen Lehrwerken (konkret Stufen international oder die beiden em-Lehrwerke) visuelle Hilfen für die Orientierung im Lehrbuch fanden - Orientierungsschwierigkeiten, und zwar offensichtlich deshalb, weil diese Lehrbücher nicht die traditionelle Organisation ${ }^{1}$ aufwiesen. Auch nach einer Besprechung der im Lehrbuch benutzten Organisation wurde der Aufbau weiterhin als ungewöhnlich empfunden.

Daneben waren auch manche Themen der Texte in kommunikativen Lehrwerken von deutschen Verlagen nicht für unsere Gruppe relevant, weil die Bücher offenbar

1 Damit ist ein Aufbau gemeint, bei dem am Anfang jeder Lektion ein synthetischer Text steht, dann eine Vokabelliste und Grammatikerklärungen folgen und grammatikalische Übungen den Hauptteil der Lektion bilden. 
eher für fremdsprachige Lernende im Zielland bestimmt sind. Für unser Kulturmilieu waren dies z. B. Themen wie die Problematik der Türken in Deutschland, die auch in verschiedenen Texten mit Alltagsproblematik (Wohnen, Einkaufen oder Familie u. ä.) eine wichtige Rolle spielen, Gespräche auf dem Arbeitsamt oder spezifische Themen wie das Bierfest in München u. ä.

Außerdem bevorzugen die meisten unserer Studierenden aus dieser Studienform systematische Grammatikvermittlung und -wiederholung gegenüber einem pragmalinguistischen Ansatz. Es handelt sich wahrscheinlich um das Problem, dass das Lernziel bei unseren Studenten/-innen nicht nur die kommunikative Beherrschung der Sprache ist, sondern auch abstraktes Wissen vom System der Sprache umfasst. Als zukünftige Lehrer und Lehrerinnen werden unsere Studierenden später nämlich das sprachliche Vorbild für ihre Schüler sein und deshalb ist es wichtig, dass sie auf jeden Fall korrektes Deutsch lernen. Das Lehrbuch sollte also möglichst viele Übungen enthalten, die zum korrekten Deutsch führen und bei denen die Studierenden möglichst wenige Fehler machen. In den neuen Lehrbüchern, z. B. in denjenigen vom Hueber Verlag (Tangramm u. a.), herrscht demgegenüber eher die Tendenz vor, die Lernenden zum freien Umgang mit der Sprache anzuregen, auch wenn sie dabei Fehler machen. Man muss aber andererseits auch eingestehen, dass ein völliges Eingehen auf die Präferenzen der Studierenden in dieser Hinsicht kontraproduktiv sein könnte. Würde das Lernen an der Universität wieder in die Bahnen eines bloßen Memorierens von Regeln und Merksätzen zurückspringen, dann würde dies wiederum zu einem rein deduktiven Unterricht an den allgemeinbildenden Schulen führen. Deswegen möchten wir das Lernen der Studierenden um autonome und moderne Elemente bereichern. Einen entsprechenden Effekt erwarten wir von der Benutzung des Lernbegleiters „Sprachenportfolio“, aber auch das kurstragende Lehrwerk sollte hier zu einer veränderten Denkweise beitragen.

Unserer Annahme nach weist zwar das von den Studierenden gewählte Lehrwerk Nëmecky súsmèvem nově eine übersichtliche Form und systematische grammatikalische Beschreibungen mittels der Muttersprache auf, die darin enthaltenen Übungen sind jedoch wenig abwechslungsreich und es fehlen Lösungen, ${ }^{1}$ die die Selbstkontrolle ermöglichen würden. Obwohl dieses Lehrwerk eine Gruppe von Studierenden ausgewählt hat, glauben andere, dass auch negative Seiten zu finden sind.

Von einigen Studierenden wurde beispielsweise die visuelle Seite des Lehrbuches als nicht genug motivierend empfunden. Das Bildmaterial beeinflusst die Wahrnehmung und das Layout beeinflusst die Motivation für das eigentliche Lernen. Einen Nachteil dieses Lehrwerks sehen wir auch darin, dass im Vergleich zu den neuen Lehrwerken der deutschen Verlage keine ergänzenden Materialien im Internet zu finden sind und keine Tipps und explizit formulierten Ziele für das

1 Es gibt nur ein extra Übungsheft mit grammatischen Übungen auf der Basis „Drillübungen“, Transkriptionen der Hörtexte und Lösungen zu den Übersetzungen. 
selbstständige Lernen geboten werden. Es fehlen auch Übungen, die dem Benutzer die eigenen Lernprozesse bewusst machen würden, und gar keine Übungen werden zu den soziolinguistischen und pragmatischen Komponenten der kommunikativen Kompetenz angeboten. Außerdem ist auch die Thematik der Lese- und Hörtexte nicht immer aktuell und authentisch genug. Aus diesen Gründen suchen wir ein anderes und aktuelleres Lehrwerk für den oben beschriebenen Studiengang. Gemäß unseren Erfahrungen ist es aber immer wichtig, das Lehrwerk in Unterricht zu erproben, weil sich erst dann in allen Details zeigt, ob das Lehrmaterial den Anforderungen gerecht werden kann. Unserer Meinung nach wäre es möglich, in zwei parallelen Kursen kurstragend ein neues und das alte Lehrwerk einzusetzen und zu beobachten, wie der Lernprozess beeinflusst wird und was das Lehrwerk zu Erfolg oder Misserfolg des Unterrichts beiträgt.

In dem Zusammenhang mit der Aufgabe, ein neues und für das Studienprogramm geeignetes Lehrwerk zu finden, stellen sich uns einige Fragen:

- Welche Themen sind authentisch und bei jungen Erwachsenen gefragt?

- Welche Problemfelder hängen eng mit dem humanistisch und pädagogisch orientierten Studium an der PdF zusammen?

- Sollte der Unterricht unbedingt mit einem Lehrwerk realisiert werden oder kann er auch lehrwerkunabhängig verlaufen?

Dies sind unseres Erachtens einige der wichtigsten Fragen, die beantwortet werden sollten, bevor wir uns für ein neues Material entscheiden.

\section{Themen und Problemfelder für junge Lerner}

Im Sprachenportfolio des Lehrstuhls haben wir schon untersucht, welche Themenbereiche Studierende während des bisherigen Sprachenlernens in welcher Intensität geübt haben ${ }^{1}$. Es hat sich gezeigt, dass die persönlichen und alltäglichen Themen im bisherigen Studienverlauf der Respondenten intensiv geübt wurden, die Fachproblematik wie Arbeitswelt, Ökologie, technischer Fortschritt oder auch interkulturelle Themen wie das Zusammenleben in einer sprachlich, kulturell und ethnisch vielfältigen Gesellschaft oder etwa auch die Beziehungen zwischen den Generationen dagegen eher selten behandelt wurden. In diesem Zusammenhang sind natürlich solche Themen und Themenaspekte geeignet, die im jeweiligen Erfahrungs- und Interessenshorizont der Studentinnen und Studenten liegen, zugleich aber auch zu vergleichenden landeskundlichen Fragestellungen herausfordern. Beata HOCKICKOVA und Rolf STEHLE $(1997,17)$ empfehlen, dass

1 Schriftlich wurden die Ergebnisse noch nicht präsentiert, nur mündlich mit den Respondenten diskutiert. Das am meisten im Unterricht bearbeitete Thema ist das Thema „Ich und meine Familie“, gefolgt von „Freizeit und Hobbys“ und „Urlaub und Reisen“. 
der Weg zur aktiven Auseinandersetzung mit der Lebenswirklichkeit im anderen Land die Einblicke in die Lebensweisen und -bedingungen von Menschen verschiedener sozialer Gruppen und unterschiedlichen Alters umfassen sollte. Ihre Werte und Normen, wie auch die entsprechenden Gegebenheiten werden dann mit den eigenen verglichen, es sollten Einsichten in die jeweiligen gesellschaftlichen Tatsachen gewonnen werden und Schlussfolgerungen für die eigene Situation und das eigene Verhalten abgeleitet werden. Auf diese Weise können z. B. auch mehrere Themen verbunden und zugleich interkulturell und motivierend bearbeitet werden. Für eine solche Vorgehensweise müsste nun eine konkrete Form und Methode gesucht werden.

\section{Die kommunikative Kompetenz der Lehramtsstudierenden}

Natürlich sollten relevante Themen auch stark mit dem akademischen Leben bzw. der späteren pädagogischen Tätigkeit der Lerner zusammenhängen.

Die kommunikative Kompetenz von zukünftigen Lehrern wäre natürlich nicht allein mit Hilfe von allgemeinen Kann-Beschreibungen im Sinne von Deskriptoren bestimmter Fertigkeiten zu definieren. Sicher ließen sich auch spezifische KannBeschreibungen definieren, wo dann das Berufsspezifische jeweils situativ mit Beispielen verdeutlicht werden müsste. Welche sprachliche Kompetenzen ein Lehrer haben sollte, hat Cornelia Gick von der Universität Fribourg aufgelistet (GICK: 2004). Die sprachlichen Kompetenzen werden im Lehrerprofil dabei beispielhaft unter den „Sozialen Kompetenzen“ und unter „Kommunikationsfähigkeit“ aufgeführt:

\section{Ad 1. Sozialkompetenz}

Fremdwahrnehmung:

$>$ Der zukünftige Lehrer/die Lehrerin kann Gruppenprozesse wahrnehmen und positive Prozesse stützen bzw. negative Prozesse auffangen.

$>$ Sie/er hat Erfahrung mit Fremd- und Selbstwahrnehmung und kann daraus Schlüsse ziehen und in eigenes Verhalten und Handeln umsetzen.

Kooperationskompetenz:

$>$ Der Student kennt verschiedene Formen der Zusammenarbeit (Alleinverantwortung für bestimmte Teile, Mitverantwortung z. B. im TeamTeaching) und Möglichkeiten der Rollenteilung (Lehrer - Schüler).

Sie/er kann Verantwortung übernehmen aber auch delegieren.

$>$ Sie/er ist bereit mit Studierenden aus anderen Fachgebieten zusammenzuarbeiten.

$>$ Sie/er ist bereit und fähig mit Institutionen(Schule, Fortbildungsinstitutionen) zusammenzuarbeiten. 
Kommunikationsfähigkeit:

$>\mathrm{Er} /$ sie kann klare Anweisungen geben.

$>\mathrm{Er} /$ sie kann sich klar mitteilen und aufmerksam zuhören.

$>\mathrm{Er} /$ sie hat Erfahrung mit Feed-back und ist bereit, Kritik zu formulieren und zu akzeptieren.

$>\mathrm{Er} /$ sie kann seine Absichten/Ziele klar kommunizieren.

Konfliktfähigkeit:

> Sie/er kennt das Konfliktpotential von Gruppen und kann Konfliktsituationen frühzeitig wahrnehmen, interpretieren und situationsgerecht darauf reagieren.

$>$ Sie/er ist bereit, aus Konflikten zu lernen.

$>$ Sie/er ist „konfliktresistent“.

Wir müssen bedenken, dass sich die Kommunikation im Unterricht im möglichst umfangreichen Maße in der Fremdsprache abspielen sollte.

\section{Ad 2. Lehrersprache und Lehrerverhalten}

> Stimmlage, Tonhöhe, Sprachniveau;

$>$ Haltung;

$>$ die Position des Sprechers im Klassenraum;

$>$ Verwendung von Gesten/Zeichen- und Gebärdensprache;

$>$ Konventionalisierung von para- und nonverbalen Zeichen;

$>$ Sich-Versichern, dass die syntaktische/morphologische/lexikale Auswahl adäquat ist

$>$ Sich-Versichern, dass die Kommunikation von Angesicht zu Angesicht stattfinden kann

> Lehrmethoden, die mehrere Sinnesmodalitäten ansprechen, verwenden.

\section{Kommunikationsfähigkeit im Lehrerberuf}

Für berufsspezifische Beschreibungen von sprachlichen Profilen müssten also auch berufsspezifische Situationen und Verwendungszwecke beschrieben werden, die nicht unbedingt nur Kann-Beschreibungen auf den höchsten Niveaus beinhalten. Im Falle eines Lehrers können das auch Kann-Beschreibungen unterer Niveaus sein (wie B1 und B2), wobei zusätzlich andere Faktoren ins Spiel kommen, die es zu berücksichtigen gilt.

Für uns wäre jetzt interessant zu beschreiben, was im Bereich „Lehrersprache“ schon auf den Niveaus B1 und B2 enthalten ist und was auf den höheren Niveaus hinzugelernt werden sollte. Im Zusammenhang mit der Lehrwerksuche zeigt sich hier eine Möglichkeit, die Kann-Beschreibungen als Unterlage zu nehmen und den 
Unterricht mit der Szenario-Technik ${ }^{1}$ ohne ein gedrucktes Lehrbuch (das nie völlig den Lernbedürfnissen unserer Studierenden angepasst werden kann) im Sinne des konstruktivistischen und ganzheitlichen Lernens zu entwerfen. Dies würde bedeuten, dass Deskriptoren der adäquaten Referenzniveaus und Deskriptoren der möglichen Szenarien der Unterrichtssprache und Unterrichtsproblematik als Grundlage für elektronisch entwickelte Materialien dienen würden, die dann im Unterricht mit Studierenden in einer Diskussion als Szenarien weiter modifiziert und präzisiert würden. Eine Liste von denkbaren Szenarien, die im Unterrichtsgeschehen zu erwarten wären, können wir zur Spezifizierung des Wortschatzes und möglichen Sprachhandlungen hier anführen (vgl. Online: http://home.t-online.de/home/ kfmaas/homepage.html [abgerufen am 2. 2. 2008]):

\section{Strukturierung des Unterrichtsgeschehens:}

1. Vorstellung, Begrüßung, Verabschiedung;

2. Unterrichtsorganisation (Sitzplan, Klassenzimmer, Gruppierung);

3. Disziplin (Ruhe, Aufmerksamkeit, Unterbinden von Störungen, Ermunterung, Lob, Tadel);

4. Stundenablauf (Stundeneröffnung, Fehlerkorrektur, Hausaufgaben, Stundenschließung);

5. Gelenkstellen des Unterrichts (mündlicher Unterricht: Aussprache, Nachsprechen, Fragen-Antworten, Rollenspiel, situative Übungen, Konversation, Diskussion, Nacherzählung, Lesen, schriftliche Arbeiten, EinsetzErgänzungs-, Umformungsübungen, freie Aufgaben u. a.);

6. Medien (auditive, visuelle, audiovisuelle);

7. Tätigkeiten.

In den einzelnen Szenarien wären dann nähere Spezifizierungen der einzelnen linguistischen Komponenten notwendig, wie sich etwa am Beispiel eins „informativen Gesprächs“ zeigen lässt. Ein solches Gespräch müsste zunächst nach dem kommunikativen Ziel charakterisiert werden. Wichtig ist weiter, dass der Aufbau des Textes im Voraus geplant wird und dass die einzusetzenden sprachlichen Mittel (zu unterscheiden nach grammatikalischen, lexikalischen und phonetischen Mitteln) und auch evtl. mögliche nonverbale Elemente dem Sprecher bekannt sind.

1 Laut Wikipedia wird dieser Begriff in der Fremdsprachendidaktik als ein Kernthema bezeichnet, das für die Lernenden Ausgangspunkt einer Lernerfahrung wird. Der Begriff wird häufig im Kontext des als „Fremdsprachenwachstum“ bezeichneten Lernkonzepts verwendet (online: www. wikipedia.de [abgerufen am 2. 7. 2008]). Es kommt zum Einsatz von Szenarien, die erwartete (oder auch verbotene) Kommunikationsabläufe beschreiben. Sie sind eine erste, weit verbreitete Methode für die Beschreibung des Verhaltens und bei der Festlegung der Anforderungen an das Verhalten und deren Analyse (vgl. GIESE online: http://wwwcs.upb.de/cs/ag-schaefer-static/Lehre/ Lehrveranstaltungen/). 
Diese Überlegungen zum Einsatz der Szenario-Methode stellen nur einen ersten Versuch dar, wie wir das Problem hinsichtlich des geeigneten Lehrmaterials betrachten bzw. lösen könnten. Allerdings bleibt noch eine ganze Reihe von Fragen ungeklärt, z. B. die genaue Definition der Lerninhalte und die Frage nach der Rolle der Grammatik. Es fehlen schlüssige Aussagen über Textsorten, Arbeitsformen, Vermittlungs- und Lernverfahren sowie entsprechende Lernerfolgskontrollen. Aus diesen Gründen bleiben wir dabei, den Unterricht mit einem Lehrwerk zu realisieren.

Aus unseren Erfahrungen können wir aber auch sagen, dass sich die Meinungen und Gewohnheiten der Studierenden entwickeln, genauso wie die Methodik des Fremdsprachenunterrichts an diversen Mittelschulen. Für das kommende Semester planen wir daher eine neue Untersuchung zu den Präferenzen der aktuellen Gruppe von Studierenden. Auch wenn kein Lehrwerk genau auf die Unterrichtsziele der Lehramtsstudierenden zugeschnitten ist und in idealer Weise den Unterrichtszielen entspricht, werden wir uns für ein Lehrwerk für junge Erwachsene entscheiden, weil wir glauben, dass das Vorhandensein eines Lehrwerks als Stütze für das Studium von den Studierenden als positiv empfunden wird. Die Themen des Lehrbuches können dann mit der Szenario-Methode ergänzt und den Deskriptoren des Sprachenportfolios, das für unsere Studierenden als ein Instrument für die Selbstevaluation des Studiums dienen sollte, angepasst werden. Ohnehin sind diese international gültigen Deskriptoren in vielen neueren Lehrwerken bereits in das Unterrichtsmaterial integriert.

\section{Zusammenfassung:}

Spielt das Lehrwerk im Fremdsprachenunterricht eine bedeutende oder nur eine untergeordnete Rolle? Welche Argumente und Kriterien sind bei der Lehrwerkauswahl wichtig und relevant? Solche und ähnliche Fragen sind die Grundlage unserer Überlegungen, die es dokumentieren, wie wir bei einer konkreten Lehrwerkauswahl vorgegangen sind. Bei der Entscheidung, welches Unterrichtsmaterial aus der gegenwärtigen Lehrmaterialproduktion ausgewählt werden sollte, spielten eine wichtige Rolle auch Fragen, was der Unterrichtende und die Lerner von diesem Medium erwarten und wie weit sie den eigentlichen Unterricht von dem methodischen Konzept dieses Materials beeinflussen lassen. Der Beitrag beschreibt Erfahrungen mit einem Lehrwerk von tschechischen und einem von deutschen Autoren.

Schlüsselwörter:

Lehrwerk - Fremdsprachenunterricht - Lehrwerkauswahl - Lehrmaterialproduktion methodisches Konzept 


\section{Literatur:}

BIMMEL et al. (2003): Bimmel Peter et al. Deutschunterricht planen. Arbeit mit Lehrwerklektionen. München: Goethe-Institut, 2003.

BRYCHOVÁ (2007): Brychová, Alice. Lehrwerkanalyse und Vergleich mit Deskriptoren des Europäischen Fremdsprachenportfolios, in: Marta, Pallová (Hg.): Der moderne Unterricht des Deutschen als Fremdsprache. Olomouc: Univerzita Palackého v Olomouci, 2007, S. 46-50.

DEUTSCH AKTIV NEU (1988): Neuner Gerhard et al. Deutsch aktiv Neu. Ein Lehrwerk für Erwachsene. Lehrerhandreichnungen 1A. Berlin/München: Langenscheidt, 1988.

DRMLOVÁ, et al. (2003): Drmlová, Daniela et al. Německy súsmèvem nově. Plzeň: Fraus, 2003.

GICK (2004): Gick, Cornelia. Einstiege ins Europäische Sprachenportfolio. Einige Ideen aus der Praxi für die Praxis. Didaktischer Beitrag, in: Babylonia, 2004, Nr. 2., S. 19-24.

EM-BRÜCKENKURS (1998): Perlmann-Balme, Michaela/Schwalb, Susanne/Weers, Dörte. Em Brückenkurs Deutsch als Fremdsprache für die Mittelstufe. Ismaning: Max Hueber Verlag, 1998. 